\title{
Ethics and Information Technology (ETIT)
}

DOI: http://doi.org/10.26480/etit.02.2020.104.108

ISBN: 978-1-948012-17-1

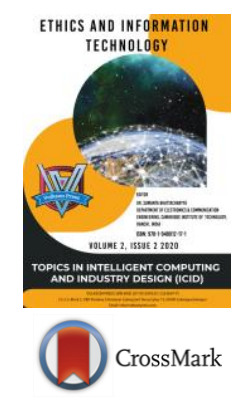

\section{FAKE NEWS DETECTION USING SUPERVISED LEARNING METHOD}

\author{
Swapnesh Jain ${ }^{\mathrm{a}}$, Ruchi Patel ${ }^{\mathrm{b}}$, Shubham Guptac, Tanu Dhoot ${ }^{\mathrm{d}}$ \\ aDepartment of Computer Science and Engineering, Medicaps University, Indore - 453331, India \\ ${ }^{b}$ Assistant Professor, Computer Science and Engineering, Medicaps University, Indore - 453331, India \\ cDepartment of Computer Science and Engineering, Medicaps University, Indore - 453331, India \\ dDepartment of Computer Science and Engineering, Medicaps University, Indore - 453331, India \\ *Corresponding Author Email: ruchi.patel@medicaps.ac.in
}

This is an open access article distributed under the Creative Commons Attribution License CC BY 4.0, which permits unrestricted use, distribution, and reproduction in any medium, provided the original work is properly cited.

\section{ARTICLE DETAILS}

\section{Article History:}

Received 25 October 2020

Accepted 26 November 2020

Available online 03 December 2020

\begin{abstract}
With the advent of social media, information has never been more accessible than today. But as much as it is accessible, there is a flood of information at the time where virtually everyone is a content creator. In this age where data is the new oil, the amount of data produced every day on social media has caused certain troubles too. One of the most notorious trouble thus created is that of "fake news". While social media has proven to be a voice of the previously voiceless and has enabled those to dismantle old one-sided narratives but on the other hand, it has also enabled people to monger fear, incite violence and spread misinformation. The most recent effect of fake news can be seen in the violence happening in New Delhi, India, where a systematic spread of misinformation led to riots against the majority community of India. In this paper, a comparative study has been made for the efficiency of various models in correctly identifying fake news along with their true positives and true negatives. The aim here is to apply count vector and tf-idf vector to four different machine learning methods such as Naïve Bayes, Logistic Regression, Random Forest and XGBoost on two different datasets namely Kaggle and LIAR. Based on the results obtained XGBoost along with count vector gave the highest accuracy in predicting fake news.
\end{abstract}

\section{KEYWORDS}

Count Vector, Fake News, Logistic Regression, Naïve Bayes, Random Forest, Tf-Idf Vector, True Positives, True Negatives, XGBoost.

\section{INTRODUCTION}

With the information revolution now being a real thing and virtually every person with a smartphone in hand and a working internet connection being a content creator everyone has to deal with a new menace of fake news almost every day with social media being its breeding ground. With more and more people being introduced to social media every day and it is a great influence on the opinion-forming process of the people, there are people with vested agendas who want to use these information as a means to propel their agenda.

This has led to the creation of many websites that publish articles containing half-truths or full lies. Some websites publish fake news almost exclusively to push propaganda (mostly political) to influence people in a certain way. The fake news industry is a global issue as well as a challenge for the world with many established media houses also losing credibility over the years after being repeatedly caught in fake news.

Many scientists believe that this problem can be dealt by using Machine Learning techniques with Artificial Intelligence. Hence this paper describes a comparative study of four most popular machine learning methods to identify and determine which method produces the best results while detecting fake news.

\section{RELATED WORK}

Fake news is a major challenge. Most challenging part of fake news detection is the detection of deceptive languages which is done by statistical methods. This issue becomes even more serious while dealing with reviews obtained from interviews on television, social media posts like those on Facebook and Twitter. Based on a study, combined sentiment analysis with network metadata to detect fake news. He trained Random Forest classifier which gave him the f1-score of more than $88 \%$. He also designed a scrapping tool to gather news related articles from different sources (Shrestha, 2018). Other research from Korea University coauthored a paper on article abstraction in which they created a factual database by collecting obvious facts of human's decisions. Their system search for the articles related to the news in their factual database to verify whether the news is reliable or not (Kim and Jeong, 2019).

A recent study implemented deep neural network for detecting fake news which include different machine learning models along with different deep learning models which evaluate their performance in identifying fake news (Kaliyar, 2018). Recent work include an integrated web service model that accepts news input or URL from the user which then checks for the truth level of the news (Mokhtar et al., 2019). Also in others study have focused on the detection of fake news by training the Naive Bayes classifier which was then tested on Facebook posts (Granik and Mesyura, 2017) Their model achieved classification accuracy of $74 \%$ on the test data. In other studies work like evaluation of different machine learning methods using tf-idf and probabilistic context-free grammar (Gilda, 2017), fake news detection on social media networks to filter out sites with false and misleading news (Aldwairi and Alwahedi, 2018), and hybrid text 
classification to deal with the classification of fake news were carried out (Kaur et al., 2019).

\section{MeTHOdOLOGIES}

For classification problem many different supervised learning techniques are present such as Naïve Bayes, Decision Trees, Support Vector Machine, Gradient Descent, K-Nearest Neighbors, K-Fold cross validation, Neural Networks, etc. Some of these popular techniques have been used in this study which are as follows:

\subsection{Naïve Bayes (NB)}

Naive Bayes algorithm is a simple but an efficient technique for the construction of classifiers. It consists of models that label problem instances as classes, which are represented as feature-vectors. There is not just a single algorithm to train all such classifiers, but there exists a family of algorithms which all are based on a common principle. All NB classifiers work on the assumption that for a given class variable the value of a feature does not depend upon the value of any other feature. Despite their naive and primitive design and seemingly oversimplified assumptions, NB classifiers have been known to function quite well in many complex scenarios that exist in the real-world. An advantage of NB classifier is that only a small number of training data is required to make an estimation of the parameters that would be necessary for classification.

\subsection{Logistic Regression (LR)}

Logistic Regression algorithm is applied when the dependent or the target variable is categorical in nature. In its basic form LR is a statistical model which makes uses of a logistic function for modelling a binary dependent variable. Models based on analogues technique that use a different kind of sigmoid function instead of using the logistic function can also be considered for logistic regression, one such example is the probity model. The characteristic feature that defines the logistic model is that when value of one of the independent variable increases multiplicatively, the probability of the second variable gets scaled at a constant rate, where each independent variable has its own parameter; this feature generalizes the odds ratio for a binary dependent variable also.

\subsection{Random Forest (RF)}

As the name implies, Random forest is a model that consists of numerous individual and independent decision trees that function simultaneously as an ensemble. Each individual trees in the RF returns a class as a prediction and the class that attains the highest number of votes becomes the prediction of the entire model. The distinguishing feature of RF is that when a fairly large number of independent trees work together, they outperform any other standalone model. The trees also enforce canopy effect like that of a real forest and protect each other from their respective individual errors (Understanding Random Forest, 2020).

\subsection{Extreme Gradient Boosting (XGBoost)}

Extreme Gradient Boosting (herein after referred to as XGBoost) is an ensemble machine learning algorithm that is based on the concept of decision trees and works on the framework of gradient boosting. In such prediction problems that deal with the data in the unstructured form (images, text, etc.) artificial neural networks tend to outdo the performance of all other algorithms and frameworks working with similar problems. However, while dealing with small-to-medium scale structured or tabular data, algorithm based on decision tree concept are considered to be the most suited model and are widely recommended for this purpose (Morde et al., 2020) XGBoost is one such gradient boosting library that is highly optimized and distributed and is designed to be highly efficient, flexible and portable.

\section{Proposed Solution}

This section consists of important steps for detecting fake news using different machine learning methods, which are as follows:

Step 1: Two different datasets, Kaggle and Liar, are gathered to implement each method.

Step 2: Then cleaning techniques such as Tokenization, Stemming and removing punctuation marks, tags, and stop-words are used on each data set to clean them.

Step 3: After cleaning, two different pre-processing techniques such as CountVectorizer (count of terms in vector) and term frequency-inverse document frequency (tf-idf) are used on each dataset to build the vocabulary of the count vectors.

Step 4: The methods then are trained using Naive Bayes, Logistic regression, Random Forest and XGBoost.

Step 5: Then performance was evaluated for these different machine learning methods and compared using their accuracy and confusion matrix.

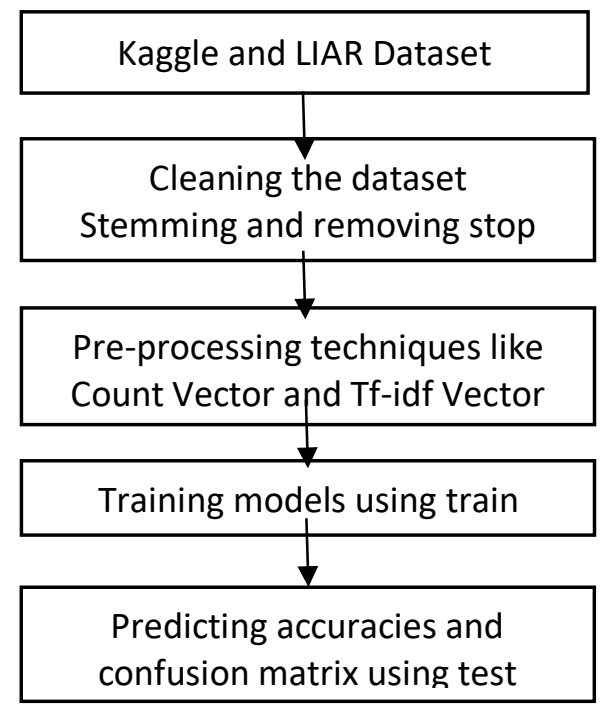

Figure 1: Flowchart of Proposed Solution

In the above proposed solution, steps 2 and 3 are important steps because these steps are not only normalizing the dataset but also converting the textual news data into numerical vectors as these machine learning methods can be trained using numerical data only. In the step 2 the dataset is first cleaned because when the dataset is gathered from different sources they are in raw format which is not acceptable by the methods. In the step 3 CountVectorizer is used to count the frequency of each word occurred in the news. TF-IDF is also used for information retrieval to determine the importance of individual terms in the set of text documents. TF-IDF's value increases proportionately with the increase in occurrence of the given word in a given document but often falls down with the frequency of the words in the corpus.

TF-IDF can be computed as the product of term frequency and inverse document frequency. Term frequency can be computed as the ratio of number of times a term appears in a document to the total number of terms in the document and Inverse Document Frequency can be computed as the log of ratio of number of documents to the number of documents that contain the word.

Once the textual data is converted into the numerical vectors (using CountVectorizer and TF-IDF) the methods are trained using these vectors so that the output of the test dataset can be predicted to compare the accuracies between these four different methods to determine their efficiency. Also a confusion matrix is generated which classifies these news as positive or negative to evaluate their performance.

In the confusion matrix shown in table 1, rows represent the number of classifications predicted by the model, while the columns represent the number of actual classifications in the test data.

\begin{tabular}{lll}
\hline & \multicolumn{2}{l}{ Table 1: Confusion Matrix } \\
\hline & Actual Positive & Actual Negative \\
\hline Predicted Positive & True Positives (TP) & False Positives (FP) \\
Predicted Negative & False Negatives (FN) & True Negatives (TN) \\
\hline
\end{tabular}

Where, TP is defined as actual real news which are correctly predicted as real,

FP is defined as actual false news which is incorrectly predicted as real, FN is defined as actual real news which is incorrectly predicted as false, and

TN is defined as actual false news which is correctly predicted as false. Accuracy is the ratio of sum of predictions that are correctly classified by the model to the total number of samples. Accuracy can be computed using the equation (1).

Accuracy $=\frac{T P+T N}{T P+T N+F P+F N}$

\section{EXPERIMENTAL SETTINGS}




\subsection{Datasets}

To implement each method two different datasets are gathered for this projects.

The first dataset is of Kaggle which includes 16,548 human labeled news articles in the train set described by five columns namely 'id', 'title', 'author', 'text' and 'label' (categorized as 0, indicating real news and 1, indicating fake news). The test dataset of Kaggle consists of 4,137 news articles described by four columns namely 'id', 'title', 'author' and 'text'. This test set contains news without label. And the submit dataset of Kaggle consists of same 4,137 news articles, as of test dataset, described by two columns namely 'id' and 'label' but with label to compute the accuracy of each method.

Second dataset is of LIAR which includes 10,240 human labeled news articles in the train set described by two columns namely 'statement' and 'label' (categorized as true and false). The test dataset of LIAR consists of 2,551 human labeled news articles described by two columns namely 'statement' and 'label' to compute the accuracy of each model.

\subsection{Pre-processing}

First both the datasets are observed to look for what type of data each methods are dealing with. But upon closely observing datasets it was found that both the Kaggle and LIAR datasets contain missing values and incomplete news articles. But Kaggle dataset along with missing values and incomplete news articles contain news from different languages also. So to increase the efficiency of each models these missing values along with the news articles which are incomplete and of different languages are eliminated. The text field plays an important role in labelling and therefore text field containing blank columns have also been deleted.

After eliminating these type of data all the special characters (or say punctuation marks and tags) along with the stop-words are removed. The stop-words are those words that do not contribute much in predicting whether the news is real or fake, instead they just introduce confusion to each model. Some of these commonly used stop-words are "a", "the", "of", "I", "you", "it", "and", etc. Rather machine would not want these words taking up space in the database, or taking up valuable processing time. Therefore eliminating them will improve the efficiency of the model. After the soft-words are removed, stemming was carried out to reduce the words to their roots.

The research has been carried out using Python language because it provides a great choice of libraries and packages to carry out most of the tasks to build machine learning models. Also Python has a great community support because most of the programmers contribute to help each other.

\section{RESULT AND ANALYSIS}

The model was trained and tested for both the datasets. In this project two techniques for pre-processing, namely 'CountVectorizer' and 'TF-IDF', and four methods of machine learning (discussed in Methodologies) are used and confusion matrix along with accuracies are obtained and tabulated.

\subsection{Experimental Result on Kaggle Dataset}

\begin{tabular}{llll}
\hline \multicolumn{3}{c}{ Table 2: Confusion Matrix when CountVectorizer was used as pre- } \\
processing technique on Kaggle dataset
\end{tabular}

Table 2 describes in detail the labels obtained from various prediction methods and also the extent of correctness. Count Vectorizer along with various prediction methods was applied on Kaggle dataset to obtain this table.

With Naïve Bayes classifier, 3489 correct and 368 incorrect predictions are obtained. With Logistic Regression, 3680 correct and 177 incorrect predictions are obtained. On applying Random Forest 3529 correct and 328 incorrect predictions are obtained. Finally while applying XGBoost 3716 correct and 141 incorrect predictions are obtained.

\begin{tabular}{ll}
\multicolumn{2}{c}{ Table 3: Accuracy table for CountVectorizer technique on Kaggle } \\
dataset
\end{tabular}

Table 3 contains the consolidated data for accuracy of various prediction methods with CountVectorizer on Kaggle dataset in percentage point terms.

Model obtained 90.45\% accuracy using Naïve Bayes, 95.41\% accuracy using Logistic regression, $91.49 \%$ accuracy using Random Forest and $96.34 \%$ accuracy using XGBoost.

\begin{tabular}{llll}
\multicolumn{4}{c}{ Table 4: Confusion Matrix when TF-IDF was used as pre-processing } \\
technique on Kaggle dataset \\
\hline Classification Algorithm & Label & $\begin{array}{l}\text { Actual } \\
\text { Positive }\end{array}$ & $\begin{array}{l}\text { Actual } \\
\text { Negative }\end{array}$ \\
\hline Naïve Bayes & $\begin{array}{l}\text { Predicted } \\
\text { Positive }\end{array}$ & 2016 & 20 \\
& $\begin{array}{l}\text { Predicted } \\
\text { Negative }\end{array}$ & 511 & 1310 \\
Logistic Regression & $\begin{array}{l}\text { Predicted } \\
\text { Positive }\end{array}$ & 1954 & 82 \\
Random Forest & $\begin{array}{l}\text { Predicted } \\
\text { Negative }\end{array}$ & 107 & 1714 \\
Predicted & 1962 & 74 \\
XGBoost & $\begin{array}{l}\text { Positive } \\
\text { Predicted } \\
\text { Negative } \\
\text { Predicted }\end{array}$ & 226 & 1595 \\
\hline $\begin{array}{l}\text { Positive } \\
\text { Predicted } \\
\text { Negative }\end{array}$ & 89 & 81 \\
\hline
\end{tabular}

Table 4 describes in detail the labels obtained from various prediction methods and also the extent of correctness. TF-IDF Vectorizer along with various prediction methods was applied on Kaggle dataset to obtain this table.

With Naïve Bayes classifier, 3326 correct and 531 incorrect predictions are obtained. With Logistic Regression, 3668 correct and 189 incorrect predictions are obtained. On applying Random Forest 3557 correct and 300 incorrect predictions are obtained. Finally while applying XGBoost 3687 correct and 170 incorrect predictions are obtained.

\begin{tabular}{ll}
\multicolumn{2}{c}{ Table 5: Accuracy table for TF-IDF technique on Kaggle dataset } \\
\hline Classification Algorithms & Accuracy \% \\
\hline Naïve Bayes & 86.23 \\
Logistic Regression & 95.09 \\
Random Forest & 92.22 \\
XGBoost & $\mathbf{9 5 . 5 9}$ \\
\hline
\end{tabular}

Table 5 contains the consolidated data for accuracy of various prediction methods with TF-IDF Vectorizer on Kaggle dataset in percentage point terms.

Model obtained $86.23 \%$ accuracy using Naïve Bayes, 95.09\% accuracy using Logistic regression, $92.22 \%$ accuracy using Random Forest and $95.59 \%$ accuracy using XGBoost.

6.2 Experimental Result on LIAR Dataset 


\begin{tabular}{llll}
\hline \multicolumn{4}{c}{ Table 6: Confusion Matrix when CountVectorizer was used as pre- } \\
processing technique on LIAR dataset
\end{tabular}

Table 6 describes in detail the labels obtained from various prediction methods and also the extent of correctness. Count Vectorizer along with various prediction methods was applied on LIAR dataset to obtain this table.

With Naïve Bayes classifier, 1567 correct and 984 incorrect predictions are obtained. With Logistic Regression, 1525 correct and 1026 incorrect predictions are obtained. On applying Random Forest 1573 correct and 978 incorrect predictions are obtained. Finally while applying XGBoost 1570 correct and 981 incorrect predictions are obtained.

\begin{tabular}{ll}
\multicolumn{2}{c}{ Table 7: Accuracy table for CountVectorizer technique on LIAR } \\
dataset
\end{tabular}

Table 7 contains the consolidated data for accuracy of various prediction methods with CountVectorizer on LIAR dataset in percentage point terms. Model obtained $61.42 \%$ accuracy using Naïve Bayes, 59.78\% accuracy using Logistic regression, $61.66 \%$ accuracy using Random Forest and $61.54 \%$ accuracy using XGBoost.

\begin{tabular}{|c|c|c|c|}
\hline Classification Algorithm & Label & $\begin{array}{l}\text { Actual } \\
\text { Positive }\end{array}$ & $\begin{array}{l}\text { Actual } \\
\text { Negative }\end{array}$ \\
\hline \multirow[t]{2}{*}{ Naïve Bayes } & $\begin{array}{l}\text { Predicted } \\
\text { Positive }\end{array}$ & 366 & 803 \\
\hline & $\begin{array}{l}\text { Predicted } \\
\text { Negative }\end{array}$ & 220 & 1162 \\
\hline \multirow[t]{2}{*}{ Logistic Regression } & $\begin{array}{l}\text { Predicted } \\
\text { Positive }\end{array}$ & 531 & 638 \\
\hline & $\begin{array}{l}\text { Predicted } \\
\text { Negative }\end{array}$ & 352 & 1030 \\
\hline \multirow[t]{2}{*}{ Random Forest } & $\begin{array}{l}\text { Predicted } \\
\text { Positive }\end{array}$ & 515 & 654 \\
\hline & $\begin{array}{l}\text { Predicted } \\
\text { Negative }\end{array}$ & 324 & 1058 \\
\hline \multirow[t]{2}{*}{ XGBoost } & $\begin{array}{l}\text { Predicted } \\
\text { Positive }\end{array}$ & 479 & 690 \\
\hline & $\begin{array}{l}\text { Predicted } \\
\text { Negative }\end{array}$ & 326 & 1056 \\
\hline
\end{tabular}

Table 8 describes in detail the labels obtained from various prediction methods and also the extent of correctness. TF-IDF Vectorizer along with various prediction methods was applied on LIAR dataset to obtain this table.

With Naïve Bayes classifier, 1528 correct and 1023 incorrect predictions are obtained. With Logistic Regression, 1561 correct and 990 incorrect predictions are obtained.

On applying Random Forest 1573 correct and 978 incorrect predictions are obtained. Finally while applying XGBoost 1535 correct and 1016 incorrect predictions are obtained.

\begin{tabular}{ll}
\multicolumn{2}{c}{ Table 9: Accuracy table for TF-IDF technique on LIAR dataset } \\
\hline Classification Algorithms & Accuracy \% \\
\hline Naïve Bayes & 59.89 \\
Logistic Regression & 61.19 \\
Random Forest & $\mathbf{6 1 . 6 6}$ \\
XGBoost & 60.17 \\
\hline
\end{tabular}

Table 9 contains the consolidated data for accuracy of various prediction methods with TF-IDF Vectorizer on LIAR dataset in percentage point terms.

Model obtained 59.89\% accuracy using Naïve Bayes, 61.19\% accuracy using Logistic regression, $61.66 \%$ accuracy using Random Forest and $60.17 \%$ accuracy using XGBoost.

This study has used two pre-processing algorithms and four prediction methods in all permutations on two datasets namely Kaggle and LIAR While testing the permutation on Kaggle dataset it was found that the highest accuracy was achieved when CountVectorizer technique was applied and XGBoost prediction method was used. The accuracy achieved by this model was $96.34 \%$.

The same set of permutation was used to predict fake news on LIAR dataset. The highest accuracy was achieved when CountVectorizer technique was applied and Random forest prediction method was used. The accuracy achieved by this model was $61.66 \%$.

\section{CONCLUSION AND FuturE WORK}

With the proliferation of social media, more and more people are gaining access to news from unconventional sources like social media rather than traditional main stream media. This has led to mushrooming of fake news platforms all over the world.

Therefore this paper analyses various text pre-processing techniques and classification methods that can predict whether a news is fake or not. In this experiment it was found that XGBoost classifier with CountVectorizer technique achieved the highest of accuracy, therefore it can be used to predict whether a news is fake or real.

In the future this model can be extended to sentiment based model and also algorithms like Recursive Neural Networks (RNN) can be applied to further improve the efficiency. This project can be extended further to include fact-checking and deep syntax analysis, as well as recommending similar credible articles. Convolutional Neural Network (CNN) can be used on image type of news to detect whether they are real news or misleading news.

\section{ACKNOWLEDGEMENTS}

We would like to express our deepest gratitude to Honourable Chancellor, Shri R. C. Mittal, Medicaps University, who has provided us with every facility to successfully carry out this project, and our profound indebtedness to Prof. (Dr.) Sunil K. Somani, Vice Chancellor, Medicaps University, whose unfailing support and enthusiasm has always boosted up our morale. We also thank Prof. (Dr.) D. K. Panda, Dean Engineering, Medicaps University, for giving us a chance to work on this project. We would also like to thank our Head of the Department, Prof. (Dr.) Suresh Jain, Medicaps University, for his continuous encouragement for betterment of the project. We express our heartfelt gratitude to our Project Guide, Dr. Ruchi Patel, Asst. Professor, Department of Computer Science \& Engineering, Medicaps University, without whose continuous help and support this project would not have been possible.

\section{REFERENCES}

Aldwairi M., Alwahedi A., 2018. Detecting Fake News in Social Media Networks, The 9th International Conference on Emerging Ubiquitous Systems and Pervasive Networks (EUSPN 2018), Procedia Computer Science, Abu Dhabi, 141, 215-222.

Gilda S, 2017. Notice of Violation of IEEE Publication Principles: Evaluating machine learning algorithms for fake news detection, IEEE 15th Student Conference on Research and Development (SCOReD), Putrajaya, 110-115.

Granik M., Mesyura V., 2017. Fake news detection using naive Bayes classifier, IEEE First Ukraine Conference on Electrical and Computer Engineering (UKRCON), Kiev, 900-903. 
Kaliyar R.K., 2018. Fake News Detection Using A Deep Neural Network, 4th International Conference on Computing Communication and Automation (ICCCA), Greater Noida, India, 1-7.

Kaur P., Boparai R. S., Singh D., 2019. Hybrid Text Classification Method for Fake News Detection, International Journal of Engineering and Advanced Technology (IJEAT), 8(5), 2388-2392.

Kim K., Jeong C., 2019. Fake News Detection System using Article Abstraction, 16th International Joint Conference on Computer Science and Software Engineering (JCSSE), Chonburi, Thailand, 209-212.
Mokhtar M. S., Jusoh Y. Y., Admodisastro N., Pa N. C., Amruddin A. Y., 2019. Fakebuster: Fake News Detection System Using Logistic Regression Technique In Machine Learning, International Journal of Engineering and Advanced Technology (IJEAT), 9(1), 2407-2410.

Morde V., XGBoost Algorithm: Long May She Reign! Medium, 2020.

Shrestha M, 2018. Detecting Fake News with Sentiment Analysis and Network Metadata, Earlham college, Richmond.

Understanding Random Forest, Medium, 2020.

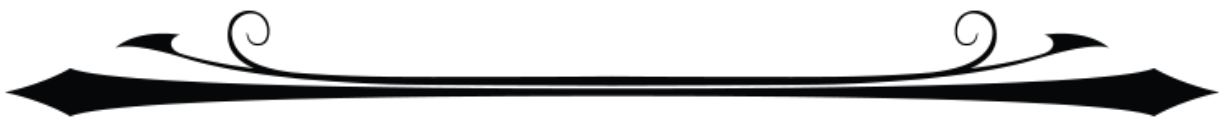

